\title{
Unresectable Leiomyosarcoma
}

National Cancer Institute

\section{Source}

National Cancer Institute. Unresectable Leiomyosarcoma. NCI Thesaurus. Code C132147.

A leiomyosarcoma which is not amenable to surgical resection. 